\title{
Genotoxicity of Microbial Volatile Organic Compounds
}

\author{
Daisuke Nakajima, ${ }^{*, a}$ Ruri Ishii, ${ }^{a}$ Shiho Kageyama, ${ }^{a}$ Yoshiki Onji, ${ }^{b}$ Shigeru Mineki, ${ }^{c}$ \\ Nobuhisa Morooka, ${ }^{d}$ Kosuke Takatori, ${ }^{e}$ and Sumio Goto ${ }^{a}$
}

${ }^{a}$ Research Center for Material Cycles and Waste Management, National Institute for Environmental Studies, 16-2 Onogawa, Tsukuba, Ibaraki 305-8506, Japan, ${ }^{b}$ Air Environment Division, Nara Prefectural Institute for Hygiene and Environment, 57-6 Ohmori-cho, Nara, Nara 630-8131, Japan, 'Faculty of Science and Technology, Tokyo University of Science, 2641 Yamazaki, Noda-shi, Chiba 2788510, Japan, ${ }^{d}$ Faculty of Home Economics, Koriyama Women's University, 3-25-2 Kaisei, Koriyama, Fukushima 963-8503, Japan,

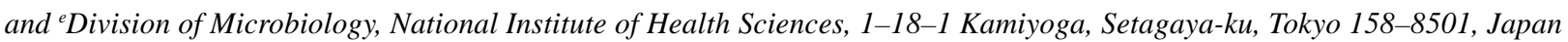

(Received November 5, 2005; Accepted January 10, 2006)

\begin{abstract}
Luminescent and light absorption ити tests were used to investigate the genotoxicity of microbial volatile organic compounds (MVOCs), which have been reported to occur in conjunction with the growth of filamentous fungi. Investigation of 20 types of MVOC samples confirmed the SOS-inducing activity of 1-octen-3-ol, 2-methyl1-propanol, 2-heptanone, 3-octanol, 1-pentanol, 1-butanol, 2-methyl-1-butanol, 3-methyl-1-butanol, 3-methyl-2butanol, 3-octanone, 2-hexanone, 2-butanone, 3-methyl-2-butanone, 2-pentanol, ethyl isobutyrate, and terpinen-4ol. Of these materials, 3-methyl-2-butanone and 3-methyl-2-butanol, which were positive in both the luminescent and light absorption ити tests, were clearly shown also to be mutagenic based on the results of the Ames test. Each of these 20 MVOCs is known to be produced by microorganisms commonly detected in indoor environments, and long-term exposure could be a health hazard.
\end{abstract}

Key words — microbial volatile organic compound, fungi, umu test, Ames test, mutagenicity

\section{INTRODUCTION}

There are many types of indoor air pollutants. Examples include volatile organic compounds (VOCs), such as formaldehyde and toluene, which have drawn attention in recent years as causes of sick building syndrome; phthalate esters (plasticizers); agents used to treat wood, such as termite-proofing agents and fire retardants; combustion exhaust gases in conjunction with heating and cooking; and even agents such as mold spores, mites, pet fur, and pollen, which are causes of allergies. Most recently, microbial VOCs (MVOCs) produced by microorganisms have been added to the list. There have been reports of more than 100 types of chemical compounds produced by several species of microorganims, ${ }^{1-3)}$ as well as reports of MVOCs being detected in rooms with severe mold damage. De-

*To whom correspondence should be addressed: Research Center for Material Cycles and Waste Management, National Institute for Environmental Studies, 16-2 Onogawa, Tsukuba, Ibaraki 305-8506, Japan. Tel.: +81-29-850-2984; Fax: +81-29-8502849; E-mail: dnakaji@nies.go.jp spite reports of various chemical analyses of MVOCs in the air from indoor environments, few reports have discussed the actual damage to health caused by MVOCs or their toxicity. To date, reports on the health effects of MVOCs have been limited to demonstrating the possibility that they cause nasal inflammation ${ }^{4)}$ and a study using the microculture tetrazolium assay and methylene blue assay to examine general toxicity in human lung epithelial cells (A549). ${ }^{5)}$ To the best of our knowledge, the only report to address genotoxicity specifically is that of Kreja and Seidel, ${ }^{6}$ who subjected human lung carcinoma epithelial A549 cells, V79 Chinese hamster fibroblasts, and human peripheral blood $\mathrm{pB}$ cells to the comet assay. Because various microorganisms are present in ordinary living environments at various concentrations, there is the possibility of human exposure to MVOCs. Exposure from indoor air can be generally considered long-term exposure, and even at low concentrations there is a possibility of health risks that should not be ignored. Therefore in this study we performed $u m u$ tests with microorganisms to investigate the SOS-inducing activity of representative MVOC samples. We report the results 
not only for the light absorption umu test, but also for the more sensitive luminescent $u m u$ test, which we additionally used to investigate genotoxicity.

\section{MATERIALS AND METHODS}

Reagents and Test Materials — The following 20 types of MVOC samples were tested: 2butanone, 2-methyl-1-propanol, ethyl isobutyrate, 2heptanone, and 3-octanone (Wako Pure Chemical Industries, Osaka, Japan, special grade); 2-hexanone and 2-nonanone (Wako Pure Chemical Industries, grade 1); terpinen-4-ol (Wako Pure Chemical Industries, highly pure); 3-methyl-2-butanone, 2-pentanol, 3-methyl-1-butanol, 1-pentanol, and 1-hexanol (Nacalai Tesque, Kyoto, Japan, special grade); 2methyl-1-butanol, 1-decanol, and 1-butanol (Nacalai Tesque, grade 1); 2- $n$-pentylfuran and 1-octen-3-ol (Lancaster Synthesis, Lancashire, U.K., highly pure); and 3-octanol and 3-methyl-2-butanol (Tokyo Kasei Kogyo, Tokyo, Japan, highly pure). All of these compounds have been reported to be produced by filamentous fungi..$^{2,3,7-9)}$ Sample dilution was performed with both dimethyl sulfoxide (DMSO), (Dojindo, Kumamoto, Japan) for luminescent measurement and methanol (Wako Pure Chemical Industries) for testing agricultural chemical residue.

Luminescent umu Test _ In the luminescent ити microplate method, luminescent genes luxA-E extracted from the luminescent bacterium Vibro fisheri are joined downstream from the ити $\mathrm{D}, \mathrm{C}$ gene of plasmid pSK1002 to construct plasmid pTL210.10) Salmonella typhimurium (S. typhimurium) TL210, which is a phenotypic transformation of S. typhimurium TA1535, is used in the plasmid. After overnight culturing of the bacterial strain, DMSO was mixed into and dispersed through the bacterial suspension to create a final concentration of $7 \%$; the frozen material was used on each test day. Tryptone-glucose-ampicillin (TGA) medium was used to dilute the frozen and cultured bacteria and for the test bacterial suspension. The TGA medium was prepared with $1 \%$ bacto-tryptone, $0.5 \%$ $\mathrm{NaCl}, 0.2 \%$ glucose, and ampicillin. The luminescent $и т и$ microplate method was performed as follows. An S. typhimurium TL210 strain of frozen bacteria was thawed and mixed with TGA medium in an Erlenmeyer flask in a 1-to-25 dilution. The suspension underwent shaking culture in an incubator at $37^{\circ} \mathrm{C}$ at $120 \mathrm{rpm}$ for $2 \mathrm{hr}$. Optical density at $600 \mathrm{~nm}\left(\mathrm{OD}_{600}\right)$ was diluted to 0.1 with TGA me- dium. DMSO or methanol was used to dilute test materials and environmental samples. ${ }^{11)}$ A 96-well, clear-bottomed, black plate (Matrix Technologies, Hudson, NH, U.S.A.) was used; $4 \mu$ of sample solution was added to each well. Next, $200 \mu \mathrm{l}$ of prepared bacterial suspension was mixed and left to stand in an incubator at $30^{\circ} \mathrm{C}$. Immediately after the bacterial suspension was mixed, the luminescence was measured with a Luminescenser-JNR AB2100 (Atto, Tokyo, Japan). Turbidity at $595 \mathrm{~nm}$ was measured with a Wallac 1420 ARVOsx plate reader (Perkin Elmer, Wellesley, MA, U.S.A.), and the number of live bacteria estimated. The shortest time between measurement periods was once per $20 \mathrm{~min}$, and the longest approximately $5 \mathrm{hr}$. In addition, when measuring luminescence, an estimated value for $1 \mathrm{sec}$ per well was sought. When genotoxicity and mutagenicity testing is done in vitro, the mammalian metabolic system is included by adding rat liver S9 mix. However, when S9 mix is added to luminescent $и т и$ tests, the value of the control solvent is reduced, and attention is required to make a determination of positive or negative, particularly for samples with a weak SOS response. ${ }^{10,12)}$ Therefore S9 mix was not added in the current study.

Light Absorption umu Test ${ }^{11,13)}$ — The light absorption $и т и$ test was performed using the $S$. typhimurium strain TA1535/pSK1002. The frozen preserved bacterial strain was rapidly dissolved, inoculated in luria-bertani (LB) medium (1\% bactotryptone, $0.5 \% \mathrm{NaCl}, 0.5 \%$ yeast extract), and cultured by undergoing shaking culture at $37^{\circ} \mathrm{C}$ at $120 \mathrm{rpm}$ for $12 \mathrm{hr}$. The culture suspension was diluted 100 times with TGA medium, and shaking culture continued for an additional $2 \mathrm{hr}$. The bacterial suspension obtained and the test materials were put into a microplate, sealed, and after thorough stirring with a plate mixer, underwent shaking culture at $37^{\circ} \mathrm{C}$ at $900 \mathrm{rpm}$ for 2 or $4 \mathrm{hr}$. The number of live bacteria in the bacterial suspension was then estimated based on the $\mathrm{OD}_{595}$. Ten microliters of bacterial suspension was transferred to each of the holes in another 96-well microplate and diluted with $140 \mu \mathrm{l}$ of $\mathrm{Z}$ buffer solution. A Z buffer solution of 2mercaptoethanol was added immediately before. Ten microliters $(4 \mathrm{mg} / \mathrm{ml})$ of chlorophenol red- $\beta$-Dgalactopyranoside (CPRG) was added to this. After culturing for $30 \mathrm{~min}$ at $37^{\circ} \mathrm{C}, 100 \mu \mathrm{l}$ of $1 \mathrm{~mol} / \mathrm{l} \mathrm{so}$ dium bicarbonate aqueous solution was added to stop coloration. Then $\beta$-galactosidase activity was measured based on $\mathrm{OD}_{570}$ (umu unit $=\mathrm{OD}_{570} / \mathrm{OD}_{595}$ ). Ames Test ${ }^{14)} \_$S. typhimurium TA98 and 
TA100 ${ }^{15)}$ were used as the test strains. To prevent solvent vaporation, we used the screw-capped test tube for preincubation. To sterilized screw-capped test tubes containing the bacterial suspension and the DMSO solution of the test substances, we added either S9 mix (+S9) or sodium phosphate buffer $0.1 \mathrm{~mol} / \mathrm{l}(-\mathrm{S} 9)$. After preincubation at $37^{\circ} \mathrm{C}$ for $20 \mathrm{~min}$, soft agar was added and mixed, and the mixture spread on the plates. After culturing for $48 \mathrm{hr}$ at $37^{\circ} \mathrm{C}$, the number of revertant colonies was counted. The S9 mix was prepared with freeze-dried material containing a set of cofactors and S9 solution (S9/Cofactor A Set for Ames Tests; Oriental Yeast Co., Tokyo, Japan). The sample solutions, including the solvent control, were diluted with DMSO to seven different strengths. The test used two plates for each strength. The positive control for $+\mathrm{S} 9$ tests was benzo[ $a]$ pyrene $(\mathrm{BaP})$, and for $-\mathrm{S} 9$ tests, 2-(2furyl)-3-(5-nitro-2-furyl)acrylamide (AF2). Microscopic examination of plate surfaces was done as needed to determine inhibited bacterial growth.

Judgment of Results — Determination of each bioassay was done for samples with a good dose response: samples for which the number of revertant colonies was 1.5 to 2 times that of spontaneous revertant colonies were deemed pseudopositives, and those that were 2 times or more positive. It is often observed that dose-response curves immediately decrease at high doses in bioassays such as the umu test or Ames test. This is because potent cytotoxicity occurs. As a result, although the response is not positive, a comparatively strong dose-response curve is often seen, unlike the case of a negative response. Therefore not only positive results but also pseudopositive results are indicated in this report.

\section{RESULTS AND DISCUSSION}

The dose-response curve from the luminescent ити test exhibits good dose dependency in the case of 3-methyl-2-butanol (Fig. 1). In this example, because the luminescence is more than double that of the control solvent, the sample was determined to be positive for SOS-inducing activity. Among the MVOCs examined in the current study, their influence on bacterial growth differed greatly, resulting in some cases in which turbidity was extremely low at MVOC concentrations above a certain amount. Accordingly, the concentration in the samples was appropriately reduced, and testing was done in a range in which the growth inhibition of the bacteria
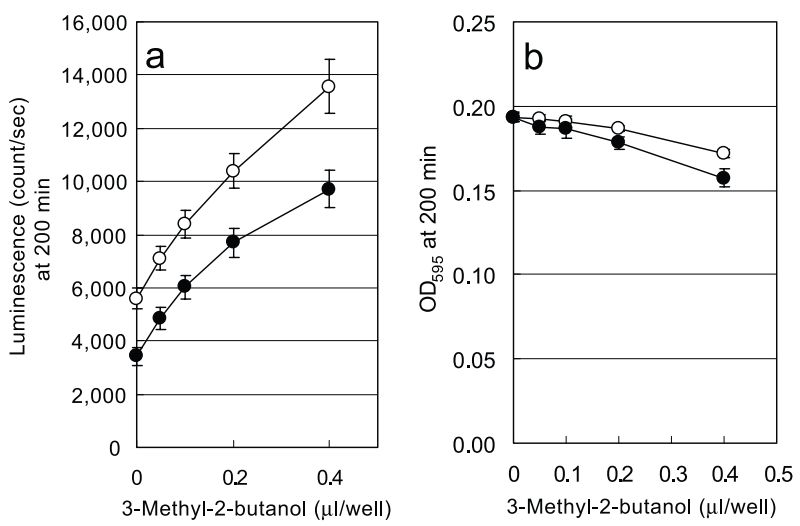

Fig. 1. Eample of a Dose-Response Curve in the Luminescent ити Test (3-Methyl-2-butanol)

a, Luminescence; b, light absorption at $595 \mathrm{~nm}$; $\bigcirc$, dilution with DMSO;, dilution with methanol.

Table 1. Response of MVOCs in the Luminescent umu Test

\begin{tabular}{|c|c|c|c|}
\hline & \multirow[t]{2}{*}{ Compound } & DMSO & $\mathrm{MeOH}$ \\
\hline & & \multicolumn{2}{|c|}{$(\operatorname{count} / \mu 1 \cdot \sec )$} \\
\hline 1 & 1-Decanol & N.D. & N.D. \\
\hline 2 & 1-Octen-3-ol & N.D. & $(18500)$ \\
\hline 3 & 3-Octanol & $(30000)$ & 14000 \\
\hline 4 & 1-Hexanol & N.D. & N.D. \\
\hline 5 & 1-Pentanol & $(25000)$ & $(42800)$ \\
\hline 6 & 1-Butanol & 9600 & 9800 \\
\hline 7 & 2-Methyl-1-butanol & 18700 & 22900 \\
\hline 8 & 3-Methyl-1-butanol & 14600 & 18600 \\
\hline 9 & 3-Methyl-2-butanol & 19400 & 15100 \\
\hline 10 & 2-Methyl-1-propanol & 12300 & 11900 \\
\hline 11 & 2-Nonanone & N.D. & N.D. \\
\hline 12 & 3-Octanone & (17900) & 12800 \\
\hline 13 & 2-Heptanone & 28300 & 22500 \\
\hline 14 & 2-Hexanone & $(18600)$ & 23300 \\
\hline 15 & 2-Butanone & 5300 & 5300 \\
\hline 16 & 3-Methyl-2-butanone & 21000 & 32000 \\
\hline 17 & 2-Pentanol & 9800 & 14300 \\
\hline 18 & Ethyl isobutyrate & $(3600)$ & 3700 \\
\hline 19 & 2-n-Pentylfuran & N.D. & N.D. \\
\hline 20 & Terpinen-4-ol & $(44000)$ & $(30000)$ \\
\hline
\end{tabular}

did not exceed $50 \%$. Test results for the chemical compounds are summarized in Table 1. Generally, in bioassays like the Ames test in which test materials are not dissolved in water, DMSO is used as the solvent, but it is known that in the light absorption umu test, DMSO itself shows an SOS response to some extent. ${ }^{16)}$ Similar phenomena have also been observed in the luminescent $u m u$ test, but the SOS 

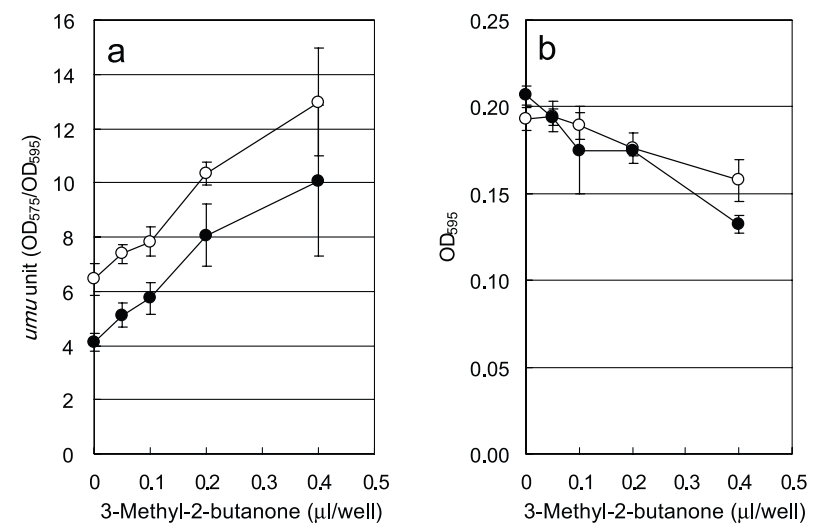

Fig. 2. Example of a Dose-Response Curve in the Light Absorption $и т и$ Test (3-Methyl-2-butanone)

a, ити unit; b, light absorption at $595 \mathrm{~nm}$; $\bigcirc$, dilution with DMSO;

, dilution with methanol.

response of methanol and similar agents is low, and it is possible that the background is suppressed to a greater extent than it is DMSO. ${ }^{11)}$ Therefore in this study tests were done using both DMSO and methanol as the solvent. When DMSO was used as the solvent, 9 of the 20 MVOCs tested positive for SOSinducing activity, and 6 displayed pseudopositive results then methanol was used as the solvent, 13 MVOCs tested positive, and 3 were pseudopositive. Of the positive MVOCs, 3-methyl-2-butanone showed the strongest activity $\left(32000 \mathrm{count} / \mathrm{sec}^{\bullet} \mu \mathrm{l}\right.$ when using methanol). Other compounds that showed comparatively strong activity included 2heptanone, 2-methyl-1-butanol, 3-methyl-2-butanol, and 3-methyl-1-butanol, followed by 3-octanone, 3octanol, 2-methyl-1-propanol, 2-hexanone, 2pentanol, 1-butanol, ethyl isobutyrate, and 2butanone.

A dose-response example from the light absorption umu test is shown in Fig. 2, and the results obtained are given in Table 2. In this test, the number of live bacteria was also estimated by measuring the turbidity of the culture medium in the wells at $595 \mathrm{~nm}$. When growth inhibition of 50\% or greater was seen, the sample was appropriately diluted and retested. In the light absorption ити test as well, both DMSO and methanol were used as dilution solvents; results are shown for each. In addition, the incubation time for both the bacterial suspension and the test materials in $и т и$ tests is usually $2 \mathrm{hr}$, but because the SOS response of each MVOC was comparatively weak, the period was extended to $4 \mathrm{hr}$ in this study. When DMSO was used as the solvent, only 3-methyl-2-butanone was positive for SOS-in-
Table 2. Response of MVOCs in the Conventional Light Absorption umu Test

\begin{tabular}{rlcc}
\hline \hline \multirow{2}{*}{ Compound } & \multicolumn{2}{c}{ DMSO } \\
\cline { 2 - 4 } & & N.D. & Mmu unit/ $\mu$ l) \\
\hline 1 & 1-Decanol & N.D. & N.D. \\
2 & 1-Octen-3-ol & N.D. & N.D. \\
3 & 3-Octanol & N.D. & N.D. \\
4 & 1-Hexanol & N.D. & N.D. \\
5 & 1-Pentanol & N.D. & N.D. \\
6 & 1-Butanol & N.D. & N.D. \\
7 & 2-Methyl-1-butanol & N.D. & N.D. \\
8 & 3-Methyl-1-butanol & (6.34) & 14.3 \\
9 & 3-Methyl-2-butanol & N.D. & N.D. \\
10 & 2-Methyl-1-propanol & N.D. & N.D. \\
11 & 2-Nonanone & N.D. & N.D. \\
12 & 3-Octanone & N.D. & N.D. \\
13 & 2-Heptanone & N.D. & N.D. \\
14 & 2-Hexanone & N.D. & N.D. \\
15 & 2-Butanone & 16.6 & 15.1 \\
16 & 3-Methyl-2-butanone & N.D. & N.D. \\
17 & 2-Pentanol & N.D. & N.D. \\
18 & Ethyl isobutyrate & N.D. & N.D. \\
19 & 2- $n$-Pentylfurane & N.D. & N.D. \\
20 & Terpinen-4-ol & p.D., not detected. Numbers in parentheses are pseudoposi- \\
\hline & tives. & &
\end{tabular}

ducing activity (3-methyl-2-butanol was a pseudopositive). When methanol was used, 3-methyl-2butanol was also positive. As in the luminescent ити test, there was a tendency for more samples to show positives when using methanol as the dilution solvent. 3-Methyl-2-butanone, which displayed the greatest activity in the luminescent $и т и$ test, also had the greatest activity in the light absorption umu test, but other MVOCs that showed comparable activity at the same level or greater than 3-methyl-2butanol in the luminescent ити test were not positive in the light absorption uти test.

It is thought that in the carcinogenesis initiation process, genetic damage occurs first, leading to mutation. The umu test is a primary screening method for detecting the SOS response induced by genetic damage. Therefore the 2 MVOCs that were positive in the light absorption umu test, 3-methyl2-butanone and 3-methyl-2-butanol, were tested for mutagenicity using the Ames preincubation method (Fig. 3). 3-Methyl-2-butanone displayed good dose dependency with both TA98 and TA100 when S9 mix was not added (-S9) and with TA98 when S9 mix was added $(+\mathrm{S} 9)$. Because under these condi- 

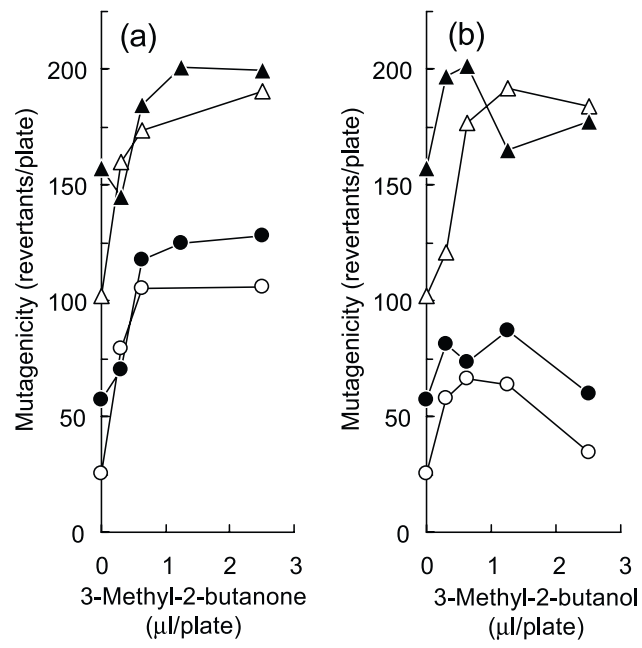

Fig. 3. Results of Ames Test of 3-Methyl-2-butanone and 3Methyl-2-butanol

(a), 3-Methyl-2-butanone; (b), 3-methyl-2-butanol; $\bigcirc$, TA98 -S9; •, TA98 +S9; $\triangle$, TA100 -S9; $\boldsymbol{\Delta}$, TA100 +S9.

tions it showed 2-fold or more revertant colonies than did the control solvent, it was determined to be positive for mutagenicity. With TA100 when S9 mix was added (+S9), an increase in the number of revertant colonies corresponding to the dose was also seen, but the number was not 2-fold that of the control solvent. With 3-methyl-2-butanol on the other hand, good dose dependency was only displayed for TA98 and TA100 when S9 mix was not added (-S9). The number of revertant colonies was 2-fold or more that of the control solvent with TA98 and 1.5-fold or more with TA100, resulting in a determination of positive and pseudopositive, respectively.

Kreja and Seidel investigated the genotoxicity of 16 types of MVOCs with the comet assay using A549, V79, and pB cells. ${ }^{6}$ According to their results, DNA damage was caused by 1-decanol, 1octen-3-ol, 2-methyl-1-propanol, and 2-heptanone, but because of the strong concentration range (near or greater than the $\mathrm{IC}_{50}$ value) for cytotoxicity, they reported that genotoxicity was not seen under the test conditions. In the current study performed with the luminescent $и т u$ test, among the MVOCs tested 1-octen-3-ol, 2-methyl-1-propanol, and 2-heptanone showed positive or pseudopositive results in concentration ranges at which $50 \%$ or more of bacterial propagation was not inhibited. However, 1-decanol had strong growth inhibitory effects on the test bacterium S. typhimurium TL210, with growth inhibition of $50 \%$ or greater seen at $4 \mathrm{nl} /$ well, and detecting the SOS response was difficult. This suggests that a similar problem exists in light absorption $и т и$ tests because detection is difficult when the sample haspotent growth-inhibitory effects on bacteria.

In the current study, in addition to the 3 MVOCs mentioned above, SOS-inducing activity was also seen in the following 13 types of MVOCs: 3-octanol, 1-pentanol, 1-butanol, 2-methyl-1-butanol, 3-methyl-1-butanol, 3-methyl-2-butanol, 3-octanone, 2hexanone, 2-butanone, 3-methyl-2-butanone, 2pentanol, ethyl isobutyrate, and terpinen-4-ol. Of these, 3-methyl-2-butanone and 3-methyl-2-butanol, which were positive in both the luminescent $u m u$ and light absorption $u m u$ tests, clearly showed mutagenicity in Ames test results. These MVOCs are reported to originate in Penicillium brevicompactum, Eurotium amstelodami, and Chaetomium globosum. ${ }^{8)}$ Also, with the addition of 2-methyl-1propanol, which showed a pseudopositive result on the luminescent $u m u$ test, these MVOCs are known to be produced during the growth of a comparatively large number of types of filamentous fungi, including Cladosporium, Aspergillus, Alternaria, and Stachybotrys. ${ }^{7)}$ All of these are fungi that are commonly detected in indoor environments. ${ }^{9}$ Therefore it is possible that these MVOCs are present in indoor environments and that long-term exposure may pose health risks. In the future, steps such as detection of known SOS-inducing MVOCs from actual environmental air and periodic monitoring are needed to understand the facts concerning contamination by such agents.

Acknowledgements The authors are grateful to researchers at Toyota Central R\&D Laboratories for supplying S. typhimurium TL210.

\section{REFERENCES}

1) Herr, C. E. W., Harpel, S., Nieden, A., Stilianakis, N. I. and Eikmann, T. F. (2002) Assessing health effects of bioaerosols measuring viable spores and microbial volatile organic compounds (MVOC) in residential air. In Indoor Air 2002: Proceedings of the International Conference on Indoor Air Quality and Climate, vol. III (Levin, H., Ed.), Indoor Air 2002, Santa Cruz, June 30-July 5, The Printing House, Inc., Stought, Wisconsin, pp. 29-34.

2) Braathen, O.-A., Schmidbauer, N., Lunder, C., Blom, P. and Mattsson, J. (2002) ORM (optimal removing of moisture from water damaged building constructions)-MVOC. In Indoor Air 2002: Proceedings of 
the International Conference on Indoor Air Quality and Climate, vol. I (Levin, H., Ed.), Indoor Air 2002, Santa Cruz, June 30-July 5, The Printing House, Inc., Stought, Wisconsin, pp. 408-413.

3) Wady, L., Bunte, A., Pehrson, C. and Larsson, L. (2002) Gas chromatography-mass spectrometry/ solid phase microextraction: a new technique for the identification of MVOCs from moldy building materials. In Indoor Air 2002: Proceedings of the International Conference on Indoor Air Quality and Climate, vol. I (Levin, H., Ed.), Indoor Air 2002, Santa Cruz, June 30-July 5, The Printing House, Inc., Stought, Wisconsin, pp. 414-419.

4) Horner, W. E. and Miller, J. D. (2003) Microbial volatile organic compounds with emphasis on those arising from filamentous fungal contaminants of buildings. ASHRAE, 4621, 215-231.

5) Kreja, L. and Seidel, H.-J. (2002) On the cytotoxicity of some microbial volatile organic compounds as studied in the human lung cell line A549. Chemosphere, 49, 105-110.

6) Kreja, L. and Seidel, H.-J. (2002) Evaluation of genotoxic potential of some microbial volatile organic compounds (MVOC) with the comet assay, the micronucleus assay and the HPRT gene mutation assay. Mutat. Res., 513, 143-150.

7) Park, J. S. and Ikeda, K. (2002) MVOC emissions from fungi in HVAC system. In Indoor Air 2002: Proceedings of the International Conference on Indoor Air Quality and Climate, vol. IV (Levin, H., Ed.), Indoor Air 2002, Santa Cruz, June 30-July 5, The Printing House, Inc., Stought, Wisconsin, pp. 335-340.

8) Schleibinger, H., Laußmann, D., Bratting, C., Mangler, B., Eis, D. and Ruden, H. (2005) Emission patterns and emission ratio of MVOC and the possibility for predicting hidden mold damage. Indoor Air, 15(Suppl. 9), 98-104.

9) Meklin, T., Potus, T., Pekkanen, J., Hyvärinen, A.,
Hirvonen, M.-R. and Nevalainen, A. (2005) Effects of moisture-damage repairs on microbial exposure and symptoms in schoolchildren. Indoor Air, 15(Suppl. 10), 40-47.

10) Tanada, K., Goto, S., Kadokami, K., Hirai, M., Imaeda, T. and Suzuki, M. (2001) Modification of umu test using the bioluminescent bacteria and application to sediments and soils. J. Environ. Chem., 11, 841-848.

11) Nakajima, D., Ishii, R., Nishimura, K., Takagi, Y., Mineki, S., Onodera, S. and Goto, S. (2005) Effects of organic solvent in luminescent umu test using $S$. typhimurium TL210. J. Environ. Chem., 15, 569574.

12) Nakajima, D., Nishimura, K., Ishii, R., Takagi, Y., Tanada, K., Mineki, S. and Goto, S. (2004) A modification of the luminescent umu test in S9 activation system. In Proceedings of China-Japan Joint Symposium on Environmental Chemistry (Yang, M. and Onodera, S., Eds.), October 21-23, Beijing, China, pp. 332-333.

13) Oda, Y., Nakamura, S., Oki, I., Kato, T. and Shinagawa, H. (1985) Evaluation of the new system (umu-test) for the detection of environmental mutagens and carcinogens. Mutat. Res., 147, 219229.

14) Yahagi, T., Nagao, M., Seino, Y., Matsushima, T., Sugimura, T. and Okada, M. (1977) Mutagenicities of $N$-nitrosoamines on Salmonella. Mutat. Res., 48, 121-130.

15) Maron, D. M. and Ames, B. N. (1983) Revised methods for Salmonella mutagenicity test. Mutat. Res., 113, 173-215.

16) Nakamura, S., Oda, Y., Shimada, T., Oki, I. and Sugimoto, K. (1987) SOS-inducing activity of chemical carcinogens and mutagens in Salmonella typhimurium TA1535/pSK1002: examination with 151 chemicals. Mutat. Res., 192, 239-246. 